\title{
On the group of alternating colored permutations
}

\author{
Eli Bagno \\ The Jerusalem College of Technology \\ Jerusalem, Israel \\ bagnoe@jct.ac.il
}

\author{
David Garber \\ Department of Applied Mathematics \\ Faculty of Sciences \\ Holon Institute of Technology \\ Holon, Israel \\ garber@hit.ac.il
}

\author{
Toufik Mansour \\ Department of Mathematics \\ University of Haifa \\ Haifa, Israel \\ toufik@math.haifa.ac.il
}

Submitted: Dec 18, 2013; Accepted: Mar 25, 2014; Published: May 9, 2014

Mathematics Subject Classifications: 20B35, 20F05

\begin{abstract}
The group of alternating colored permutations is the natural analogue of the classical alternating group, inside the wreath product $\mathbb{Z}_{r} 2 S_{n}$. We present a 'Coxeterlike' presentation for this group and compute the length function with respect to that presentation. Then, we present this group as a covering of $\mathbb{Z}_{\frac{r}{2}} \succ S_{n}$ and use this point of view to give another expression for the length function. We also use this covering to lift several known parameters of $\mathbb{Z}_{\frac{r}{2}} \succ S_{n}$ to the group of alternating colored permutations.
\end{abstract}

\section{Introduction}

The group of colored permutations, $G_{r, n}$, is a natural generalization of the Coxeter groups of types A (the symmetric group) and B (the hyperoctahedral group). Extensive research has been devoted to extending the enumerative combinatorics aspects and methods from the symmetric group to the group of colored permutations (see for example $[2,3,8,14$, $16,17]$, and many more).

It is well-known that the symmetric group $S_{n}$ has a system of Coxeter generators which consists of the adjacent transpositions:

$$
\{(i, i+1) \mid 1 \leqslant i \leqslant n-1\} \text {. }
$$


The alternating subgroup, $A_{n}$, which is the kernel of the sign homomorphism, is a wellknown subgroup of the symmetric group of index 2. A pioneering work, expanding one of the fascinating branches of enumerative combinatorics, namely, the study of permutation statistics to $A_{n}$, has been done by Roichman and Regev in [15]. They defined some natural statistics which equidistribute over $A_{n}$ and yielded identities for their generating functions.

Brenti, Reiner, and Roichman [7] dealt with the alternating subgroup of an arbitrary Coxeter group. They started by exploring Bourbaki's presentation [4, Chap. IV, Sec. 1, Exer. 9] and elaborated on a huge spectrum of extensions of the permutation statistics of $S_{n}$ to the (general) alternating group.

In this paper, we study the subgroup of $G_{r, n}$, consisting of what we call alternating colored permutations, which is the analogue of the usual alternating group $A_{n}$ in the colored permutation group. For every $n \in \mathbb{N}$ and even $r$, the mapping which sends all 'Coxeter-like' generators of $G_{r, n}$ (see the definition in Section 2 ) to -1 is a $\mathbb{Z}_{2}$-character, whose kernel is what we call here the group of alternating colored permutations, denoted by $A_{r, n}$. We present here a generalization of Bourbaki's presentation, for $r=4 k+2$, equipped with a set of canonical words, an algorithm to find a canonical presentation for each element of the group, and a combinatorial length function.

For the study of permutation statistics of $A_{n}$, Regev and Roichman [15] used a covering map from $A_{n+1}$ to $S_{n}$, which enabled them to pass parameters from $S_{n}$ to the alternating group $A_{n+1}$. In this paper, we use a similar idea, where in this time we consider the group of alternating colored permutations as a $2^{n-1}$-cover of the group of colored permutations of half the number of colors. We use this technique to shed a combinatorial flavor on our length function and to pass some statistics and their generating function to the group of alternating colored permutations.

Note that there are two additional candidates for the group of alternating colored permutations. Namely, every $\mathbb{Z}_{2}$-character of $G_{r, n}$ provides a kernel which deserves to be called a group of alternating colored permutations. A work in this direction, which gives a profound treatment to the other two non-trivial kernels, and points out the connections between the three groups, and some interesting properties of each group separately is in progress.

The paper is organized as follows. In Section 2, we gather the needed definitions on the colored permutation group, as well as some notations which we use in the sequel. A Coxeter-like presentation for the group of colored permutations, $G_{r, n}$, is presented at the end of this section. The notion of alternating colored permutations is introduced in Section 3. We present its set of generators, and show their corresponding relations. In Section 4, we present an algorithm for writing each element as a product of the generators. A detailed analysis of that algorithm yields a set of canonical words, as well as a length function. Section 5 is devoted to some technical proofs, as well as to the generating function of the length function.

In Section 6, we present the covering map and study the structure of the cosets, thereby providing a way to decompose the length function via the quotient group. The part of the 
length which varies over each coset (fiber) is called the fibral length and is studied here in a combinatorial way. Then we provide a generating function for this parameter. In Section 7, we give some examples for using the covering map for lifting parameters from the colored permutations group of half the number of colors to the group of alternating colored permutations.

\section{Preliminaries and notations}

In this section, we gathered some notations as well as preliminary notions which will be needed for the rest of the paper.

\subsection{The group of colored permutations}

Definition 1. Let $r$ and $n$ be positive integers. The group of colored permutations of $n$ digits with $r$ colors is the wreath product

$$
G_{r, n}=\mathbb{Z}_{r} \curlywedge S_{n}=\mathbb{Z}_{r}^{n} \rtimes S_{n}
$$

consisting of all pairs $(\vec{z}, \tau)$, where $\vec{z}$ is an $n$-tuple of integers between 0 and $r-1$ and $\tau \in S_{n}$. The multiplication is defined by the following rule: for $\vec{z}=\left(z_{1}, \ldots, z_{n}\right)$ and $\overrightarrow{z^{\prime}}=\left(z_{1}^{\prime}, \ldots, z_{n}^{\prime}\right)$

$$
(\vec{z}, \tau) \cdot\left(\overrightarrow{z^{\prime}}, \tau^{\prime}\right)=\left(\left(z_{1}+z_{\tau^{-1}(1)}^{\prime}, \ldots, z_{n}+z_{\tau^{-1}(n)}^{\prime}\right), \tau \circ \tau^{\prime}\right)
$$

(the operation + is taken modulo $r$ ).

Another way to present $G_{r, n}$ is as follows: Consider the alphabet

$$
\Sigma=\left\{1, \ldots, n, \overline{1}, \ldots, \bar{n}, \ldots, 1^{[r-1]}, \ldots, n^{[r-1]}\right\}
$$

as the set $\{1, \ldots, n\}$ colored by the colors $0, \ldots, r-1$. Then, an element of $G_{r, n}$ is a colored permutation, i.e., a bijection $\pi: \Sigma \rightarrow \Sigma$ satisfying the following condition: if $\pi\left(i^{[\alpha]}\right)=j^{[\beta]}$, then $\pi\left(i^{[\alpha+1]}\right)=j^{[\beta+1]}$ (the addition in the exponents is taken modulo $r$ ). Using this approach, the element $\pi=\left(\left(z_{1}, \ldots, z_{n}\right), \tau\right) \in G_{r, n}$ is the permutation on $\Sigma$, satisfying $\pi(i)=\pi\left(i^{[0]}\right)=\tau(i)^{\left[z_{\tau(i)}\right]}$ for each $1 \leqslant i \leqslant n$. For example, the element $\pi=$ $\left((2,1,0,3,0,0),\left(\begin{array}{llllll}1 & 2 & 3 & 4 & 5 & 6 \\ 2 & 1 & 4 & 3 & 6 & 5\end{array}\right)\right) \in G_{6,6}$ satisfies: $\pi(1)=2^{[1]}, \pi(2)=1^{[2]}, \pi(3)=$ $4^{[3]}, \pi(4)=3^{[0]}, \pi(5)=6^{[0]}, \pi(6)=5^{[0]}$.

For an element $\pi=(\vec{z}, \tau) \in G_{r, n}$ with $\vec{z}=\left(z_{1}, \ldots, z_{n}\right)$, we write $z_{i}(\pi)=z_{i}$, and denote $|\pi|=(\overrightarrow{0}, \tau)$. We define also $c_{i}(\pi)=r-z_{i}\left(\pi^{-1}\right)$ and $\vec{c}(\pi)=\vec{c}=\left(c_{1}, \ldots, c_{n}\right)$. Using this notation, the element $\pi=(\vec{z}, \tau)=\left((2,1,0,3,0,0),\left(\begin{array}{cccccc}1 & 2 & 3 & 4 & 5 & 6 \\ 2 & 1 & 4 & 3 & 6 & 5\end{array}\right)\right)$ satisfies $\vec{c}=(1,2,3,0,0,0)$. 
We usually write $\pi$ in its window notation (or one line notation): $\pi=\left(a_{1}^{\left[c_{1}\right]} \cdots a_{n}^{\left[c_{n}\right]}\right)$, where $a_{i}=\tau(i)$, so in the above example, we have: $\pi=\left(2^{[1]} 1^{[2]} 4^{[3]} 3^{[0]} 6^{[0]} 5^{[0]}\right)$ or just $(\overline{2} \overline{\overline{1}} \overline{\overline{4}} 365)$.

Note that $z_{i}$ is the color of the digit $i$ ( $i$ is taken from the window notation), while $c_{j}$ is the color of the digit $\tau(j)$. Here, $j$ stands for the place, whence $i$ stands for the value.

The group $G_{r, n}$ is generated by the set of generators $\mathcal{S}=\left\{s_{0}, s_{1}, \ldots, s_{n-1}\right\}$, defined by their action on the set $\{1, \ldots, n\}$ as follows:

$$
s_{i}(j)=\left\{\begin{array}{cc}
i+1 & j=i \\
i & j=i+1 \\
j & \text { otherwise }
\end{array}\right.
$$

whereas the generator $s_{0}$ is defined by

$$
s_{0}(j)=\left\{\begin{array}{lc}
\overline{1} & j=1 \\
j & \text { otherwise. }
\end{array}\right.
$$

It is well-known that the group $G_{r, n}$ has the following 'Coxeter-like' presentation with respect to the set of generators $\mathcal{S}$ (see e.g. [6]):

\section{Presentation 2.}

- $s_{0}^{r}=1$,

- $s_{i}^{2}=1$ for $1 \leqslant i \leqslant n-1$,

- $s_{i} s_{i+1} s_{i}=s_{i+1} s_{i} s_{i+1}$ for $1 \leqslant i<n$,

- $s_{i} s_{j}=s_{j} s_{i}$ for $1 \leqslant i<j<n, j-i>1$,

- $\left(s_{0} s_{1}\right)^{2 r}=1$.

\subsection{Some permutation statistics}

For $\pi \in G_{r, n}$, define the length of $\pi$ with respect to the set of generators $\mathcal{S}$ to be the minimal number of generators whose product is $\pi$. Formally:

$$
\ell(\pi)=\min \left\{r \in \mathbb{N}: \pi=s_{i_{1}} \cdots s_{i_{r}}, \text { for } i_{1}, \ldots, i_{r} \in\{0, \ldots, n-1\}\right\} .
$$

Definition 3. The length order on the alphabet

$$
\Sigma=\left\{1, \ldots, n, \overline{1}, \ldots, \bar{n}, \ldots, 1^{[r-1]}, \ldots, n^{[r-1]}\right\}
$$

is defined as follows:

$$
n^{[r-1]}<\cdots<\bar{n}<\cdots<1^{[r-1]}<\cdots<\overline{1}<1<\cdots<n
$$


Let $\sigma \in G_{r, n}$. We define:

$$
\operatorname{csum}(\sigma)=\sum_{i=1}^{n} c_{i}(\sigma)=\sum_{i=1}^{n} z_{i}(\sigma) .
$$

For $\pi \in G_{r, n}$, the inversion number, $\operatorname{inv}(\pi)$, is defined as follows:

$$
\operatorname{inv}(\pi)=|\{(i, j) \mid i<j, \pi(i)>\pi(j)\}|,
$$

where the partial order is the length order defined above.

For any $a, n \in \mathbb{N}$, let $R_{n}(a)$ be the representative of $[a] \in \mathbb{Z}_{n}$ satisfying $0 \leqslant a<n$. Moreover, assume that $r=4 k+2$ for $k \in \mathbb{N}$.

In the sequel, we will use the following operator:

Definition 4. Let $a \in \mathbb{N}$. Define:

$$
a \oslash 2=\left\{\begin{array}{cc}
R_{\frac{r}{2}}\left(\frac{a}{2}\right) & a \equiv 0(\bmod 2) \\
R_{\frac{r}{2}}\left(\frac{a+\frac{r}{2}}{2}\right) & a \neq 0(\bmod 2)
\end{array}\right.
$$

It is clear that the operator $\oslash$ commutes with the addition operation in $\mathbb{Z}_{\frac{r}{2}}$, i.e.

$$
((a+b) \oslash 2) \equiv((a \oslash 2)+(b \oslash 2))\left(\bmod \frac{r}{2}\right)
$$

\section{The group of alternating colored permutations}

The main target of this paper is the group of alternating colored permutations. We define it now. Let $\varphi$ be the function defined on the set $\mathcal{S}$ by $\varphi\left(s_{i}\right)=-1$ for any $0 \leqslant i \leqslant n-1$. It is easy to see that for even $r, \varphi$ can be uniquely extended to a homomorphism from $G_{r, n}$ to $\mathbb{Z}_{2}$, since the lengths of all the relations in Presentation 2 are even (see Lemma 1.4.1 in [5] for the corresponding proof for all Coxeter groups). so the following is well-defined:

Definition 5. Let $r$ be an even positive number. Define:

$$
A_{r, n}=\operatorname{ker}(\varphi) .
$$

The group $A_{r, n}$ is called the alternating subgroup of $G_{r, n}$.

Since $A_{r, n}$ is a subgroup of index 2, we have: $\left|A_{r, n}\right|=\frac{r^{n} n !}{2}$.

In this paper, we concentrate on the case $r=4 k+2$ where $k \in \mathbb{N}$. The other case, $r=4 k$ where $k \in \mathbb{N}$, will be treated in a subsequent paper.

We start by presenting a set of generators for $A_{r, n}$ (we prove that they indeed generate the group in Theorem 11). Define:

$$
\mathcal{A}=\left\{a_{0}, a_{1}, a_{1}^{-1}, a_{2}, \ldots, a_{n-1}\right\},
$$


where:

$$
\begin{aligned}
& a_{i}=s_{0}^{\frac{r}{2}} s_{i} \quad \text { for } 1 \leqslant i \leqslant n-1 \\
& a_{0}=s_{0}^{2} .
\end{aligned}
$$

Using Presentation 2, and the definition of the generators $a_{i}$, one can verify that the following translation relations hold in $G_{r, n}$ :

1. $s_{i} s_{j}=a_{i} a_{j}$ for $i, j \in\{2, \ldots, n-1\}$,

2. $s_{1} s_{i}=a_{1}^{-1} a_{i}$ for $i \in\{2, \ldots, n-1\}$,

3. $s_{i} s_{1}=a_{i} a_{1}$ for $i \geqslant 2$,

4. $s_{0} s_{1}=a_{0}^{\frac{r+2}{4}} a_{1}$,

5. $s_{1} s_{0}=a_{1}^{-1} a_{0}^{\frac{r+2}{4}}$

6. $a_{0}^{\frac{r}{2}}=1$,

7. $s_{0} s_{i}=a_{0}^{\frac{r+2}{4}} a_{i}$.

\section{Combinatorial algorithm for presenting elements of $\boldsymbol{A}_{r, n}$}

In this section, we introduce an algorithm which presents each element of $A_{r, n}$ as a product of the set of generators $\mathcal{A}$ of $A_{r, n}$ in a canonical way.

Let $\pi \in A_{r, n}$. We first refer to $\pi$ as an element of $G_{r, n}$ and apply the known algorithm on $\pi$ to write it as a product of elements in $\mathcal{S}$. In the second step, we translate that presentation into the set of generators $\mathcal{A}$ of $A_{r, n}$.

The algorithm for writing $\pi$ as a product of elements in $\mathcal{S}$ consists of two parts: the coloring part and the ordering part.

In the coloring part, we start from the identity element and color all the digits $i$ having $z_{i} \neq 0$. This part terminates with an ordered permutation $\sigma$ with respect to the length order. In the second part, we use only generators of the set $\mathcal{S}-\left\{s_{0}\right\}$ to arrive at $\pi$ from the ordered permutation $\sigma$.

\subsection{The coloring part}

Define: $\operatorname{Col}(\pi)=\left\{1 \leqslant i \leqslant n \mid z_{i}(\pi) \neq 0\right\}$, and $\operatorname{col}(\pi)=|\operatorname{Col}(\pi)|$. Note that the set $\operatorname{Col}(\pi)$ contains the colored digits in the image of $\pi$, (i.e. those appearing in the window notation), and not their places. We order $\operatorname{Col}(\pi)$ as follows: $\operatorname{Col}(\pi)=\left\{i_{1}<i_{2}<\cdots<i_{\operatorname{col}(\pi)}\right\}$.

We start with the identity element and color each digit $i \in \operatorname{Col}(\pi)$ by $z_{i}$ colors. This process is done according to the order of the elements in $\operatorname{Col}(\pi)$. We use the element $s_{i_{k}-1} s_{i_{k}-2} \cdots s_{1} s_{0}^{z}$ to color the digit $i_{k}$ by $z$ colors. 
Example 6. Let $\pi=\left(12^{[2]} 45^{[1]} 3^{[3]}\right) \in G_{6,5}$.

$$
(12345) \stackrel{s_{1} s_{0}^{2}}{\rightarrow}\left(2^{[2]} 1345\right) \stackrel{s_{2} s_{1} s_{0}^{3}}{\rightarrow}\left(3^{[3]} 2^{[2]} 145\right) \stackrel{s_{4} s_{3} s_{2} s_{1} s_{0}}{\longrightarrow}\left(5^{[1]} 3^{[3]} 2^{[2]} 14\right)=\sigma .
$$

The permutation $\sigma$ is an ordered permutation with respect to the length order.

\subsection{The ordering part}

For simplifying the presentation, in this part we start with $\pi$ and arrive at the ordered permutation $\sigma$, instead of continuing the algorithm from the point we have left it at the end of the coloring part.

We start by pushing the element $i_{1}=|\sigma|(1)$ in the window notation of $\pi$ to its correct place. Let $p=|\pi|^{-1}\left(i_{1}\right)$. The pushing is done by multiplying $\pi$ (from the right) by the element $u_{1}=s_{p-1} s_{p-2} \cdots s_{1}$.

Now, we continue to push the other digits of $\pi$ : for each $1<k \leqslant n-2$, if $i_{k}=|\sigma|(k)$ is located at position $p$, we use the element $s_{p-1} s_{p-2} \cdots s_{k}$ in order to push the digit $i_{k}$ to its correct place.

Example 7. We continue the previous example. Again, let $\pi=\left(12^{[2]} 45^{[1]} 3^{[3]}\right)$. The coloring part ends with the following ordered permutation:

$$
\sigma=\left(5^{[1]} 3^{[3]} 2^{[2]} 14\right)
$$

Now, we go the other way around: we start with $\pi$ and order it until we reach $\sigma$ :

$$
\begin{aligned}
\pi & =\left(12^{[2]} 45^{[1]} 3^{[3]}\right) \stackrel{s_{3} s_{2} s_{1}}{\rightarrow}\left(5^{[1]} 12^{[2]} 43^{[3]}\right) \stackrel{s_{4} s_{3} s_{2}}{\rightarrow} \\
& \rightarrow\left(5^{[1]} 3^{[3]} 12^{[2]} 4\right) \stackrel{s_{3}}{\rightarrow}\left(5^{[1]} 3^{[3]} 2^{[2]} 14\right)=\sigma .
\end{aligned}
$$

Therefore, we have:

$$
\pi=\underbrace{s_{1} s_{0}^{2} \cdot s_{2} s_{1} s_{0}^{3} \cdot s_{4} s_{3} s_{2} s_{1} s_{0}}_{\text {coloring part }} \cdot \underbrace{s_{3} \cdot s_{2} s_{3} s_{4} \cdot s_{1} s_{2} s_{3}}_{\text {ordering part }} .
$$

The algorithm described above gives a reduced word representing $\pi$ in the generators of $G_{r, n}$. This fact was proved in [2, Theorem 4.3]. The same algorithm can also be found in [17]; see also [16]. The word which was obtained in this way is called the canonical decomposition of $\pi$.

\subsection{Translation}

Now, we translate the word obtained by the algorithm described above into a word in the generators in $\mathcal{A}$ : Let $\pi \in A_{r, n}$. Use the above algorithm to write a reduced expression of $\pi$ (in the usual generators of $G_{r, n}$ ) in the form: $s_{i_{1}} s_{i_{2}} \cdots s_{i_{2 k}}$. Divide the elements of the 
reduced expression into pairs: $\left(s_{i_{1}} s_{i_{2}}\right) \cdots\left(s_{i_{2 k-1}} s_{i_{2 k}}\right)$. Now, insert $s_{0}^{\frac{r}{2}} s_{0}^{\frac{r}{2}}$ inside each pair, as follows:

$$
\begin{aligned}
\left(s_{i_{1}} s_{0}^{\frac{r}{2}} s_{0}^{\frac{r}{2}} s_{i_{2}}\right) \cdots\left(s_{i_{2 k-1}} s_{0}^{\frac{r}{2}} s_{0}^{\frac{r}{2}} s_{i_{2 k}}\right) & =\left(s_{i_{1}} s_{0}^{\frac{r}{2}}\right)\left(s_{0}^{\frac{r}{2}} s_{i_{2}}\right) \cdots\left(s_{i_{2 k-1}} s_{0}^{\frac{r}{2}}\right)\left(s_{0}^{\frac{r}{2}} s_{i_{2 k}}\right)= \\
& =a_{i_{1}}^{\varepsilon_{1}} \cdots a_{i_{2 k}}^{\varepsilon_{i_{2 k}}}
\end{aligned}
$$

where $\varepsilon_{i_{j}}=1$ if $i_{j}>1, \varepsilon_{i_{j}} \in\{ \pm 1\}$ if $i_{j}=1$, and $\varepsilon_{i_{j}} \in\left\{1, \ldots, \frac{r}{2}-1\right\}$ if $i_{j}=0$.

Example 8. We continue with $\pi=\left(12^{[2]} 45^{[1]} 3^{[3]}\right) \in A_{6,5}$ from the previous examples. As we saw, $\pi=s_{1} s_{0}^{2} s_{2} s_{1} s_{0}^{3} s_{4} s_{3} s_{2} s_{1} s_{0} s_{3} s_{2} s_{3} s_{4} s_{1} s_{2} s_{3}$. Now, we perform the translation:

$$
\begin{aligned}
\pi= & \left(s_{1} s_{0}\right)\left(s_{0} s_{2}\right)\left(s_{1} s_{0}\right)\left(s_{0} s_{0}\right)\left(s_{4} s_{3}\right)\left(s_{2} s_{1}\right)\left(s_{0} s_{3}\right)\left(s_{2} s_{3}\right)\left(s_{4} s_{1}\right)\left(s_{2} s_{3}\right)= \\
= & \left(s_{1} s_{0}^{3}\right)\left(s_{0}^{3} s_{0}\right)\left(s_{0} s_{0}^{3}\right)\left(s_{0}^{3} s_{2}\right)\left(s_{1} s_{0}^{3}\right)\left(s_{0}^{3} s_{0}\right)\left(s_{0} s_{0}^{3}\right)\left(s_{0}^{3} s_{0}\right)\left(s_{4} s_{0}^{3}\right)\left(s_{0}^{3} s_{3}\right) \cdot \\
& \cdot\left(s_{2} s_{0}^{3}\right)\left(s_{0}^{3} s_{1}\right)\left(s_{0} s_{0}^{3}\right)\left(s_{0}^{3} s_{3}\right)\left(s_{2} s_{0}^{3}\right)\left(s_{0}^{3} s_{3}\right)\left(s_{4} s_{0}^{3}\right)\left(s_{0}^{3} s_{1}\right)\left(s_{2} s_{0}^{3}\right)\left(s_{0}^{3} s_{3}\right)= \\
= & a_{1}^{-1} \mathbf{a}_{\mathbf{0}}^{\mathbf{2}} \mathbf{a}_{\mathbf{0}}^{\mathbf{2}} a_{2} a_{1}^{-1} \mathbf{a}_{\mathbf{0}}^{\mathbf{2}} \mathbf{a}_{\mathbf{0}}^{\mathbf{2}} \mathbf{a}_{\mathbf{0}}^{\mathbf{2}} a_{4} a_{3} a_{2} a_{1} a_{0}^{2} a_{3} a_{2} a_{3} a_{4} a_{1} a_{2} a_{3}= \\
= & a_{1}^{-1} a_{0} a_{2} a_{1}^{-1} a_{4} a_{3} a_{2} a_{1} a_{0}^{2} a_{3} a_{2} a_{3} a_{4} a_{1} a_{2} a_{3} .
\end{aligned}
$$

In the last equality, we cancelled some appearances of the bold-faced generator $a_{0}$, since in $A_{6,5}, a_{0}^{3}=1$.

\subsection{Analysis of the algorithm}

For analyzing the algorithm described above, we define the following sets of elements of $G_{r, n}$ and $A_{r, n}$.

\subsubsection{The coloring part}

Let

$$
C_{1}=\left\{1, s_{0}^{2}, s_{0}^{4} \ldots, s_{0}^{r-2}\right\}=\left\{1, a_{0}, \ldots, a_{0}^{\frac{r-2}{2}}\right\} .
$$

For each $1<i \leqslant n$, define for odd $i-1$ :

$$
\begin{aligned}
C_{i}^{0} & =\left\{s_{0} s_{i-1} \cdots s_{1}, s_{0} s_{i-1} \cdots s_{1} s_{0}^{2}, s_{0} s_{i-1} \cdots s_{1} s_{0}^{4}, \ldots, s_{0} s_{i-1} \cdots s_{1} s_{0}^{r-2}\right\} \\
C_{i}^{1} & =\left\{1, s_{i-1} \cdots s_{1} s_{0}, s_{i-1} \cdots s_{1} s_{0}^{3}, \ldots, s_{i-1} \cdots s_{1} s_{0}^{r-1}\right\}
\end{aligned}
$$

or, in the language of the set $\mathcal{A}$ of generators of $A_{r, n}$ :

$$
\begin{aligned}
C_{i}^{0} & =\left\{\begin{array}{l}
a_{0}^{\frac{r+2}{4}} a_{i-1} \cdots a_{1}, a_{0}^{\frac{r+2}{4}} a_{i-1} \cdots a_{1} a_{0}, a_{0}^{\frac{r+2}{4}} a_{i-1} \cdots a_{1} a_{0}^{2}, \ldots, \\
a_{0}^{\frac{r+2}{4}} a_{i-1} \cdots a_{1} a_{0}^{\frac{r}{2}-1}
\end{array}\right\} \\
C_{i}^{1} & =\left\{1, a_{i-1} \cdots a_{1}^{-1}, a_{i-1} \cdots a_{2} a_{1}^{-1} a_{0}, \ldots, a_{i-1} \cdots a_{1}^{-1} a_{0}^{\frac{r}{2}-1}\right\} .
\end{aligned}
$$

For even $i-1$, we define:

$$
\begin{aligned}
C_{i}^{0} & =\left\{s_{0} s_{i-1} \cdots s_{1} s_{0}, s_{0} s_{i-1} \cdots s_{1} s_{0}^{3}, \ldots, s_{0} s_{i-1} \cdots s_{1} s_{0}^{r-1}\right\} \\
C_{i}^{1} & =\left\{1, s_{i-1} \cdots s_{1}, s_{i-1} \cdots s_{1} s_{0}^{2}, s_{i-1} \cdots s_{1} s_{0}^{4}, \ldots, s_{i-1} \cdots s_{1} s_{0}^{r-2}\right\},
\end{aligned}
$$


or, in the language of the set $\mathcal{A}$ of generators of $A_{r, n}$ :

$$
\begin{aligned}
& C_{i}^{0}=\left\{\begin{array}{l}
\left.a_{0}^{\frac{r+2}{4}} a_{i-1} \cdots a_{1}^{-1}, a_{0}^{\frac{r+2}{4}} a_{i-1} \cdots a_{1}^{-1} a_{0}, a_{0}^{\frac{r+2}{4}} a_{i-1} \cdots a_{1}^{-1} a_{0}^{2}, \ldots,\right\} \\
a_{0}^{\frac{r+2}{4}} a_{i-1} \cdots a_{1}^{-1} a_{0}^{\frac{r}{2}-1}
\end{array}\right\} \\
& C_{i}^{1}=\left\{1, a_{i-1} \cdots a_{1}, a_{i-1} \cdots a_{1} a_{0}, a_{i-1} \cdots a_{1} a_{0}^{2}, \ldots, a_{i-1} \cdots a_{1} a_{0}^{\frac{r}{2}-1}\right\} .
\end{aligned}
$$

Define also:

$$
C_{i}=C_{i}^{0} \cup C_{i}^{1} \text { and } C_{n+1}=\left\{1, a_{0}^{\frac{r+2}{4}}\right\} .
$$

Let $\pi \in A_{r, n}$. Write $\pi$ as a product of the generators of $G_{r, n}$ in the canonical form described above.

If there is no coloring part, then $\pi \in A_{n}$ (the classical alternating group in $S_{n}$ ), so its expression contains an even number of generators from the set $\left\{s_{1}, \ldots, s_{n-1}\right\}$. We can easily make the pairing by the relations mentioned above. The length of such an expression is clearly inv $(\pi)$ (note that in this case, it does not matter whether we use the length order or the usual order).

Otherwise, we start with the coloring part. Denote by $i=i_{1}$ the smallest colored digit in the window notation of $\pi$, and by $z=z_{i_{1}}$ its color. We divide our treatment into four cases:

1. $\boldsymbol{i}-\mathbf{1}$ and $\boldsymbol{z}$ are both even: In this case, we translate $s_{i-1} \cdots s_{1}$ to $a_{i-1} \cdots a_{1}$ and $s_{0}^{z}$ to $a_{0}^{\frac{z}{2}}$, so the contribution of this sub-expression is $i-1+R_{\frac{r}{2}}\left(\frac{z}{2}\right)=i-1+R_{\frac{r}{2}}(z \oslash 2)$. We have used $a_{i-1} \cdots a_{1} a_{0}^{\frac{z}{2}} \in C_{i}$.

2. $i-1$ is even and $z$ is odd: In this case, we translate

$$
s_{i-1} \cdots s_{1} s_{0}^{z-1}
$$

to $a_{i-1} \cdots a_{1} a_{0}^{\frac{z-1}{2}} \in C_{i}$ and leave an additional generator $s_{0}$ which will be treated during the coloring of the next digit, or just before the ordering part. Note that since $\pi \in A_{r, n}$, there must be some $s_{j}, j \neq 0$, appearing right after the sub-expression $s_{i-1} \cdots s_{1} s_{0}^{z}$. In calculating the contribution of coloring the current digit (including the missing generator $s_{0}$ which will be paired later), consider the sub-expression:

$$
s_{0}^{z} s_{j}=s_{0}^{\frac{r}{2}} s_{0}^{z+\frac{r}{2}} s_{j}=a_{0}^{\frac{z+\frac{r}{2}}{2}} s_{0}^{\frac{r}{2}} s_{j}=a_{0}^{\frac{z+\frac{r}{2}}{2}} a_{j}=a_{0}^{z \oslash 2} a_{j} .
$$

Hence, $i$ contributes $i-1+R_{\frac{r}{2}}(z \oslash 2)$. We have used:

$$
a_{i-1} \cdots a_{1} a_{0}^{\frac{z-1}{2}} \in C_{i}
$$

and note that in the next colored digit, we complete the remaining $a_{0}^{\frac{r+2}{4}}$, since $z \oslash 2=\frac{z-1}{2}+\frac{r+2}{4}$. If $i$ is the last colored digit, then the term $a_{0}^{\frac{r+2}{4}} \in C_{n+1}$ will be chosen from the set $C_{n+1}$. 
3. $\boldsymbol{i}-\mathbf{1}$ and $\boldsymbol{z}$ are both odd: In this case, the sub-expression $s_{i-1} \cdots s_{2}$ will be translated to $a_{i-1} \cdots a_{2}$ and $s_{1} s_{0}^{z}$ will be written as $s_{1} s_{0}^{\frac{r}{2}} s_{0}^{z+\frac{r}{2}}=a_{1}^{-1} a_{0}^{z \oslash 2}$.

This expression contributes $i-1+R_{\frac{r}{2}}(z \oslash 2)$ to the length of $\pi$, and we have used:

$$
a_{i-1} \cdots a_{2} a_{1}^{-1} a_{0}^{z \oslash 2} \in C_{i}
$$

4. $\boldsymbol{i}-\mathbf{1}$ is odd and $\boldsymbol{z}$ is even: Here, again, the sub-expression $s_{i-1} \cdots s_{2}$ will be translated to $a_{i-1} \cdots a_{2}$ and $s_{1} s_{0}^{z-1}$ will be translated to $a_{1}^{-1} a_{0}^{\frac{z-1+\frac{r}{2}}{2}}=a_{1}^{-1} a_{0}^{(z-1) \oslash 2}$, so we use:

$$
a_{i-1} \cdots a_{2} a_{1}^{-1} a_{0}^{(z-1) \oslash 2} \in C_{i},
$$

and leave an additional generator $s_{0}$ which will be paired with some $s_{j}$ during the coloring of the next digit or just before the ordering part. In order to calculate the contribution of coloring this digit to the length of $\pi$ (including the missing generator $s_{0}$ which will be paired later), we borrow the generator $s_{j}$ appearing just after the coloring expression of the current digit: $s_{1} s_{0}^{z} s_{j}=s_{1} s_{0}^{\frac{r}{2}} s_{0}^{z+\frac{r}{2}} s_{j}$. Since we wrote: $s_{1} s_{0}^{\frac{r}{2}}=a_{1}^{-1}$, we are left with $s_{0}^{z+\frac{r}{2}} s_{j}=a_{0}^{z \oslash 2}$. The contribution in this case is again $i-1+R_{\frac{r}{2}}(z \oslash 2)$. Now, since

$$
z \oslash 2 \equiv\left((z-1) \oslash 2+\frac{r+2}{4}\right)\left(\bmod \frac{r}{2}\right)
$$

we take:

$$
a_{i-1} \cdots a_{1}^{-1} a_{0}^{(z-1) \oslash 2} \in C_{i}
$$

and the remaining $a_{0}^{\frac{r+2}{4}}$ will be taken from the next colored digit or from $C_{n+1}$ (as in case (2)).

Now, we apply the same procedure to the next colored digits, but note that there might be a situation in which the expression coloring the digit $j$ is $s_{0} s_{j-1} s_{j-2} \cdots$, due to the debt of the generator $s_{0}$ from the preceding colored digit, so the cases might be switched after converting $s_{0} s_{j}$ to $a_{0}^{\frac{r+2}{4}} a_{j-1}$.

The following example will illuminate the situation.

Example 9. Let $\pi=\left(12^{[2]} 45^{[1]} 3^{[3]}\right) \in A_{6,5}$. Then the ordered permutation is: $\sigma=$ $\left(5^{[1]} 3^{[3]} 2^{[2]} 14\right)$. We perform the coloring part:

$$
\begin{aligned}
& \text { (1) } \stackrel{s_{1} s_{0}=a_{1}^{-1} a_{0}^{2}}{\longrightarrow}\left(2^{[1]} 1354\right) \stackrel{\text { (2) } s_{0} s_{2} s_{1} s_{0}^{3}=a_{0}^{2} a_{2} a_{1}^{-1}}{\longrightarrow}\left(3^{[3]} 2^{[2]} 145\right) \rightarrow
\end{aligned}
$$

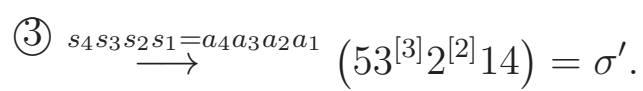

Step (1): The smallest colored digit is 2 , which has to be colored by two colors, so we are in case (4). We choose $s_{1} s_{0}=a_{1}^{-1} a_{0}^{2}$ from $C_{2}^{1}$. The additional generator $s_{0}$ will be 
treated in the next step. Note that in the calculation of the contribution of this step to the length of $\pi$ we borrow the generator $s_{2}$ from the next colored digit:

$$
s_{1} s_{0}^{2} s_{2}=s_{1} s_{0}^{3+2+3} s_{2}=\left(s_{1} s_{0}^{3}\right) s_{0}^{2}\left(s_{0}^{3} s_{2}\right)=a_{1}^{-1} a_{0} a_{2} .
$$

This expression contributes only 2 to the length of $\pi$. The generator $a_{2}$ will be counted in the next step.

Step (2): The next colored digit is 3 , and we have a debt of a generator $s_{0}$ from the previous step. Thus, we choose:

$$
s_{0} s_{2} s_{1} s_{0} s_{0} s_{0}=s_{0}^{4}\left(s_{0}^{3} s_{2}\right)\left(s_{1} s_{0}^{3}\right)=a_{0}^{2} a_{2} a_{1}^{-1} \in C_{3}^{0} .
$$

Even though $i-1=2$ is even and $z=3$ is odd (case (2)), after $s_{0} s_{2}$ in the previous step, we are actually again in case (4). Note that the expressions $a_{1}^{-1} a_{0}^{2}$ from step (1) and $a_{0}^{2} a_{2} a_{1}^{-1}$ from step (2) join together to be $a_{1}^{-1} a_{0} a_{2} a_{1}^{-1}$.

Step (3): The next colored digit is 5 . We choose:

$$
s_{4} s_{3} s_{2} s_{1}=\left(s_{4} s_{0}^{3}\right)\left(s_{0}^{3} s_{3}\right)\left(s_{2} s_{0}^{3}\right)\left(s_{0}^{3} s_{1}\right)=a_{4} a_{3} a_{2} a_{1} \in C_{5}^{1},
$$

and leave the treatment of the additional generator $s_{0}$ to the next step (in this case, to the transition between the coloring part and the ordering part, i.e. $a_{0}^{2} \in C_{6}$ ). We will elaborate on this point after describing the ordering part.

\subsubsection{The ordering part}

We turn now to the ordering part. For $1 \leqslant k \leqslant n-1$, define the sets $O_{k}$ as follows:

$$
\begin{aligned}
O_{1} & =\left\{s_{i} s_{i-1} \cdots s_{1} s_{0}^{\frac{r}{2} \varepsilon} \mid 1 \leqslant i \leqslant n-1, \quad \varepsilon \equiv i(\bmod 2)\right\} \cup\{1\}= \\
& =\left\{a_{i} a_{i-1} \cdots a_{1}^{1-2 \varepsilon} \mid 1 \leqslant i \leqslant n-1, \quad \varepsilon \equiv i(\bmod 2)\right\} \cup\{1\},
\end{aligned}
$$

and for $2 \leqslant k \leqslant n-1$ :

$$
\begin{aligned}
O_{k} & =\left\{s_{i} s_{i-1} \cdots s_{k} s_{0}^{\frac{r}{2} \varepsilon} \mid k \leqslant i \leqslant n-1, \quad \varepsilon \equiv(i+1-k)(\bmod 2)\right\} \cup\{1\}= \\
& =\left\{a_{i} a_{i-1} \cdots a_{k} \mid k \leqslant i \leqslant n-1\right\} \cup\{1\} .
\end{aligned}
$$

We start by pushing the digit $i_{1}=|\sigma|(1)$ of $\pi$ to its correct place. Let $p_{1}=\left|\pi^{-1}\right|\left(i_{1}\right)$. The pushing will be done by multiplying $\pi$ (from the right) by the element $o_{1} \in O_{1}$, where:

$$
o_{1}=\left\{\begin{array}{cc}
s_{p_{1}-1} s_{p_{1}-2} \cdots s_{1}=a_{p_{1}-1} \cdots a_{1} & p_{1}-1 \text { is even } \\
s_{p_{1}-1} s_{p_{1}-2} \cdots s_{1} s_{0}^{\frac{r}{2}}=a_{p_{1}-1} \cdots a_{2} a_{1}^{-1} & p_{1}-1 \text { is odd. }
\end{array}\right.
$$

Now, we continue to push the other digits of $\pi$ : for each $1<k \leqslant n-2$, assuming that the digit $i_{k}=\sigma(k)$ is now located at position $p_{k}$, we use the element $o_{k} \in O_{k}$ defined by:

$$
o_{k}=\left\{\begin{array}{cc}
s_{p_{k}-1} s_{p_{k}-2} \cdots s_{k}=a_{p_{k}-1} \cdots a_{k} & p_{k}-k \text { is even } \\
s_{p_{k}-1} s_{p_{k}-2} \cdots s_{k} s_{0}^{\frac{r}{2}}=a_{p_{k}-1} \cdots a_{k} & p_{k}-k \text { is odd, }
\end{array}\right.
$$

in order to push the digit $i_{k}$ to its correct place in $\pi$. Now, we have two possibilities: 
- The coloring part was completed without remainders, which means that both the coloring part and the ordering part consist of even number of $G_{r, n}$-generators. In this case, we choose $1 \in C_{n+1}$, and we have that:

$$
\pi=\gamma_{1} \cdots \gamma_{n} \cdot 1 \cdot o_{n-1}^{-1} \cdots o_{1}^{-1}
$$

where $\gamma_{i} \in C_{i}$ for $1 \leqslant i \leqslant n$, and $o_{i} \in O_{i}$ for $1 \leqslant i \leqslant n-1$.

- The coloring part has a remainder of the generator $s_{0}$. This means that the coloring part required an odd number of $G_{r, n}$-generators, and therefore the ordering part had an odd number of $G_{r, n}$-generators as well (since the sum of their lengths is even). Thus, in the ordering part, there will be a remainder of $s_{0}^{\frac{r}{2}}$, so we choose $s_{0}^{\frac{r}{2}} s_{0}=s_{0}^{\frac{r}{2}+1}=a_{0}^{\frac{r+2}{4}} \in C_{n+1}$, and we have that:

$$
\pi=\gamma_{1} \cdots \gamma_{n} \cdot a_{0}^{\frac{r+2}{4}} \cdot o_{n-1}^{-1} \cdots o_{1}^{-1}
$$

where $\gamma_{i} \in C_{i}$ for $1 \leqslant i \leqslant n$, and $o_{i} \in O_{i}$ for $1 \leqslant i \leqslant n-1$.

In both cases, we have now: $\pi=\sigma \cdot o_{n-1}^{-1} \cdots o_{1}^{-1}$, and we are done.

Example 10. We continue the previous example. Again, let $\pi=\left(12^{[2]} 45^{[1]} 3^{[3]}\right)$. After the completion of the coloring part, we have reached the permutation: $\sigma^{\prime}=\left(53^{[3]} 2^{[2]} 14\right)$.

Now, we go the other way around: we start with $\pi$ and order it to obtain $\sigma^{\prime}$ :

$$
\begin{aligned}
& \left(12^{[2]} 45^{[1]} 3^{[3]}\right) \quad(7) \stackrel{s_{3} s_{2} s_{1}=a_{3} a_{2} a_{1}^{-1} s_{0}^{3}}{\longrightarrow} \quad\left(5^{[4]} 12^{[2]} 43^{[3]}\right) \stackrel{\text { (6) } s_{4} s_{3} s_{2} s_{0}^{3}=a_{4} a_{3} a_{2}}{\longrightarrow} \\
& \rightarrow\left(5^{[1]} 3^{[3]} 12^{[2]} 4\right) \quad \stackrel{(5)}{\stackrel{s_{3} s_{0}^{3}=a_{3}}{\longrightarrow}} \quad\left(5^{[4]} 3^{[3]} 2^{[2]} 14\right) \rightarrow \\
& \text { (4) } \stackrel{s_{0}^{-4}=a_{0}^{-2}}{\longrightarrow}\left(53^{[3]} 2^{[2]} 14\right)=\sigma^{\prime} \text {. }
\end{aligned}
$$

In step (7), we push the digit 5 to its correct place with respect to the permutation $\sigma^{\prime}$. We use $s_{3} s_{2} s_{1} s_{0}^{3}=a_{3} a_{2} a_{1}^{-1} \in O_{1}$. Note that the digit 5 is bearing extra three colors. In step (6), we push the digit 3 into its place, using $s_{4} s_{3} s_{2} s_{0}^{3}=a_{4} a_{3} a_{2} \in O_{2}$. Note that the color of the digit 5 is correct again. Next, in step (5), we push 2 to its correct place using $s_{3} s_{0}^{3}=a_{3} \in O_{3}$.

Now, note that after completing step (5), we still did not arrive at $\sigma^{\prime}$, since the digit 5 has again a wrong color. On the other hand, we have a debt of a generator $s_{0}$ from the coloring part. Both problems would be solved simultaneously by using $s_{0}^{-1} s_{0}^{-3}=a_{0}^{-2} \in$ $C_{6}^{-1}$. This is exactly what we have done in step (4).

From the above analysis, we can conclude that each permutation $\pi \in A_{r, n}$ has a canonical decomposition with respect to the set $\mathcal{A}$. This is the content of the following theorem. 
Theorem 11. The set $\mathcal{A}=\left\{a_{i} \mid 0 \leqslant i \leqslant n-1\right\} \cup\left\{a_{1}^{-1}\right\}$ generates $A_{r, n}$. Moreover, for each $\pi \in A_{r, n}$, there is a unique presentation as:

$$
\pi=\gamma_{1} \cdots \gamma_{n} \gamma_{n+1} \cdot o_{n-1}^{-1} \cdots o_{1}^{-1}
$$

where $\gamma_{i} \in C_{i}$ for $1 \leqslant i \leqslant n+1$ and $o_{j} \in O_{j}$ for $1 \leqslant j \leqslant n-1$. This presentation is called the canonical decomposition of $\pi$.

Proof. Let $M$ be the Cartesian product:

$$
M=C_{1} \times \cdots \times C_{n} \times C_{n+1} \times O_{n-1} \times \cdots \times O_{1} .
$$

We start by defining a subset $L$ of $M$ which we call the set of legal vectors. A vector $\vec{\omega}=\left(\gamma_{1}, \ldots, \gamma_{n}, \gamma_{n+1}, o_{n-1}, \ldots, o_{1}\right) \in M$ is called a legal vector if it satisfies the following two conditions:

1. Let $i$ and $j$ be two indices satisfying $\gamma_{k}=1$ for all $i<k<j, \gamma_{i} \neq 1$ and $\gamma_{j} \neq 1$ (i.e. the digits $i$ and $j$ are colored, but the digits between them are not colored). If $\gamma_{i}$ ends with $s_{0}^{r-1}$, then $\gamma_{j}$ does not start with $s_{0}$.

2. Let $i$ and $j$ be two indices satisfying $\gamma_{k}=1$ for all $i<k<j, \gamma_{i} \neq 1$ and $\gamma_{j} \neq 1$. If $\gamma_{i}$ ends with $s_{1}$, then $\gamma_{j}$ starts with $s_{0}$.

We have to prove the following two claims:

(a) The algorithm associates a legal vector in $L$ to any $\pi \in A_{r, n}$. This proves the existence of the presentation.

(b) $|L|=\frac{r^{n} n !}{2}\left(=\left|A_{r, n}\right|\right)$, which implies the uniqueness of the presentation.

Claim (a) is implied immediately from the algorithm, so we pass to the proof of Claim (b). For that, we define the notions of external and internal components of a vector:

$$
\vec{\omega}=\left(\gamma_{1}, \ldots, \gamma_{n}, \gamma_{n+1}, o_{n-1}, \ldots, o_{1}\right) \in L
$$

A component $\gamma_{i} \in C_{i}$ is called external, if it ends either with $s_{0}^{r-1}$ or with $s_{1}$ (i.e. a component which imposes a restriction on the next non-trivial component), and internal otherwise. Note that $\gamma_{1}$ is always internal, since by definition, the generator $s_{1}$ does not appear in $\gamma_{1}$ and it cannot end with the expression $s_{0}^{r-1}$ since $r-1$ is odd.

For constructing an element of $L$, we start by choosing the element $\gamma_{1} \in C_{1}$, out of $\frac{r}{2}$ possibilities. Next, we choose which components will be external. Note that for each external component there are two possibilities, but each external component restricts the possibilities for the next non-trivial component (i.e. $\gamma_{i} \neq 1$ ). Next, for each internal component, we choose one out of $r-1$ possibilities.

When the coloring part is over, we have two possibilities: If we have no remainder from the coloring part, then we choose $\gamma_{n+1}=1 \in C_{n+1}$, and we have to complete the 
process by a permutation of $S_{n}$ of even length. On the other hand, if we do have a remainder, then we choose $\gamma_{n+1}=a^{\frac{r+2}{4}} \in C_{n+1}$ and we have to complete the process by a permutation of $S_{n}$ of odd length. Altogether, this contributes $n$ ! possibilities for completing the presentation.

Following the above discussion, we have that the number of legal vectors is:

$$
|L|=\frac{r}{2} \cdot\left[\sum_{i=0}^{n-1}\left(\begin{array}{c}
n-1 \\
i
\end{array}\right)(r-1)^{n-1-i}\right] \cdot n !=\frac{r \cdot n !}{2}((r-1)+1)^{n-1}=\frac{r^{n} n !}{2},
$$

as needed.

Remark 12. Note that we do not claim that the presentation described above is irreducible as it. Take for example the expression for $\pi=\left(12^{[2]} 45^{[1]} 3^{[3]}\right)$, computed in Example 8, to be:

$$
a_{1}^{-1} \mathbf{a}_{\mathbf{0}}^{\mathbf{2}} \mathbf{a}_{\mathbf{0}}^{\mathbf{2}} a_{2} a_{1}^{-1} \mathbf{a}_{\mathbf{0}}^{\mathbf{2}} \mathbf{a}_{\mathbf{0}}^{\mathbf{2}} \mathbf{a}_{\mathbf{0}}^{\mathbf{2}} a_{4} a_{3} a_{2} a_{1} a_{0}^{2} a_{3} a_{2} a_{3} a_{4} a_{1} a_{2} a_{3},
$$

which can be shortened to $a_{1}^{-1} a_{0} a_{2} a_{1}^{-1} a_{4} a_{3} a_{2} a_{1} a_{0}^{2} a_{3} a_{2} a_{3} a_{4} a_{1} a_{2} a_{3}$. On the other hand, after we cancel all the redundant appearances of $a_{0}$, we do obtain an irreducible expression, as will be proven in the next section (Corollary 21).

For $\pi \in A_{r, n}$, let $L_{A}(\pi)$ be the number of generators needed to write $\pi$ as a product of the $A_{r, n}$-generators by the algorithm.

As a consequence of the analysis of the algorithm, we have the following result:

Theorem 13. 1. Let $\pi \in A_{r, n}$, and let $\omega=s_{0}^{z_{1}} b_{1} s_{0}^{z_{2}} b_{2} \cdots s_{0}^{z_{n}} b_{n}$ where $b_{i} \in\left(\mathcal{S}-\left\{s_{0}\right\}\right)^{*}$ (i.e. a word written using generators from $\mathcal{S}-\left\{s_{0}\right\}$ ) be its canonical presentation with respect to $\mathcal{S}$. Then the translation of $\omega$ to the generators in $\mathcal{A}$ will be

$$
a_{0}^{z_{1} \oslash 2} b_{1}^{\prime} a_{0}^{z_{2} \oslash 2} b_{2}^{\prime} \cdots a_{0}^{z_{n} \oslash 2} b_{n}^{\prime}
$$

where $b_{i}^{\prime} \in\left(\mathcal{A}-\left\{a_{0}\right\}\right)^{*}$.

Moreover, if $b_{i}=s_{i_{1}} \cdots s_{i_{k}}$, then $b_{i}^{\prime}=a_{i_{1}}^{\varepsilon_{i_{1}}} \cdots a_{i_{k}}^{\varepsilon_{i_{k}}}$, where $\varepsilon_{i_{j}} \in\{ \pm 1\}$ if $i_{j}=1$ and $\varepsilon_{i_{j}}=1$ otherwise.

2. Let $\pi \in A_{r, n}$. Then:

$$
L_{A}(\pi)=\sum_{z_{i}(\pi) \neq 0}(i-1)+\operatorname{inv}(\pi)+\sum_{i=1}^{n}\left(z_{i}(\pi) \oslash 2\right) .
$$

Proof. Part (1) is straightforward from the analysis of the algorithm, so we proceed to the proof of part (2).

In [2, Theorem 4.3], the algorithm for presenting an element in $G_{r, n}$ is described (see also [17]). It is proven that for $\pi \in G_{r, n}$, the length of $\pi$, with respect to the generators in $\mathcal{S}$, is:

$$
\ell_{G_{r, n}}(\pi)=\sum_{z_{i}(\pi) \neq 0}(i-1)+\operatorname{inv}(\pi)+\sum_{i=1}^{n} z_{i}(\pi)
$$


so by part (1) we have:

$$
L_{A}(\pi)=\sum_{z_{i}(\pi) \neq 0}(i-1)+\operatorname{inv}(\pi)+\sum_{i=1}^{n}\left(z_{i}(\pi) \oslash 2\right)
$$

Another consequence of the algorithm is the following criterion for being an element in the group $A_{r, n}$ :

Theorem 14. Let $\pi \in G_{r, n}$. Then: $\pi \in A_{r, n}$ if and only if:

$$
\operatorname{csum}(\pi)+\operatorname{inv}(|\pi|) \equiv 0(\bmod 2) .
$$

Proof. By the definition, $\pi \in A_{r, n}$ if and only if $\ell_{G_{r, n}}(\pi) \equiv 0(\bmod 2)$. By the algorithm described above, $\ell_{G_{r, n}}(\pi)=\operatorname{csum}(\pi)+k$, where $k$ is the number of generators $s_{i}$, for $i \neq 0$, used in the presentation of $\pi$. On the other hand, if we remove the appearances of $s_{0}$ from the presentation of $\pi$, we get $|\pi|$. Since lengths of different presentations of the same element of $S_{n}$ have the same parity, we have that $k \equiv \operatorname{inv}(|\pi|)(\bmod 2)$, and therefore the criterion follows.

\section{The presentation of $A_{r, n}$ and its length function}

In [6], Dynkin-like diagrams were presented for the groups $G_{r, n}$. Such diagrams are based on a Coxeter-like presentation. In this section, we compute a Coxeter-like presentation for $A_{r, n}$, as well as a Dynkin-like diagram for the groups $A_{r, n}$.

\subsection{The presentation of $A_{r, n}$}

We start with the presentation of $A_{r, n}$.

Theorem 15. The set $\mathcal{A}=\left\{a_{0}, a_{1}^{ \pm 1}, \ldots, a_{n-1}\right\}$ generates $A_{r, n}$, subject to the following relations:

$$
\begin{aligned}
& \text { 1. } a_{0}^{\frac{r}{2}}=1 \text {, } \\
& \text { 2. } a_{1}^{4}=1 \text {, } \\
& \text { 3. } a_{i}^{2}=1 \text { for } i>1 \text {, } \\
& \text { 4. } a_{i} a_{j}=a_{j} a_{i} \text { for }|i-j|>1 \text { and } i, j \neq 1 \text {, } \\
& \text { 5. }\left(a_{i} a_{i+1}\right)^{3}=1 \text { for } i \geqslant 1, \\
& \text { 6. }\left(a_{0} a_{1}\right)^{2 r}=1 \text {, }
\end{aligned}
$$


7. $\left(a_{0} a_{1}^{-1}\right)^{2 r}=1$,

8. $a_{0} a_{1}^{2}=a_{1}^{2} a_{0}$,

9. $a_{1} a_{i}=a_{i} a_{1}^{-1}$ for $i>2$.

We denote by $\mathcal{R}$ the above set of relations.

Remark 16. Note that relation (5) implies $\left(a_{1}^{-1} a_{2}\right)^{3}=1$ too, and relation (8) implies $a_{1}^{-1} a_{0} a_{1}^{-1}=a_{1} a_{0} a_{1}$ and $a_{1}^{-1} a_{0} a_{1}=a_{1} a_{0} a_{1}^{-1}$.

Proof. We have already shown in Theorem 11 that $\mathcal{A}$ generates $A_{r, n}$. Here, we prove that the set $\mathcal{R}$ is a complete set of relations for $A_{r, n}$.

We imitate the idea of the proof of Proposition 2.1.1 in [7].

Consider the abstract group $A_{r, n}^{+}$generated by the elements:

$$
\mathcal{A}=\left\{a_{0}, a_{1}, a_{1}^{-1}, \ldots, a_{n-1}\right\}
$$

with $\mathcal{R}$ as the set of relations. Note that the set mapping $\alpha: \mathcal{A} \rightarrow A_{r, n}^{+}$defined by:

$$
\begin{gathered}
\alpha\left(a_{1}^{\varepsilon}\right)=a_{1}^{-\varepsilon} \text { for } \varepsilon \in\{-1,1\}, \\
\alpha\left(a_{i}\right)=a_{i} \text { for } i \in\{0,2, \ldots, n-1\},
\end{gathered}
$$

extends to a group automorphism $\alpha$ on $A_{r, n}^{+}$. Indeed, considering $A_{r, n}$ as a subgroup of $G_{r, n}, \alpha$ is the inner automorphism defined by the conjugation by $s_{0}^{\frac{r}{2}}$.

Thus, the group $\mathbb{Z}_{2}=\{1, \alpha\}$ acts on $A_{r, n}^{+}$and we have the semidirect product $A_{r, n}^{+} \rtimes \mathbb{Z}_{2}$, where the product is defined as follows:

$$
\left(x_{1} \alpha^{i}\right) \cdot\left(x_{2} \alpha^{j}\right)=x_{1} \alpha^{i}\left(x_{2}\right) \cdot \alpha^{i+j} .
$$

The semidirect product has the following presentation:

$$
A_{r, n}^{+} \rtimes \mathbb{Z}_{2}=\left\langle\alpha, a_{0}, a_{1}, a_{1}^{-1}, a_{2}, \ldots, a_{n-1} \mid \begin{array}{c}
\mathcal{R}, \alpha a_{i} \alpha=\alpha\left(a_{i}\right) \text { for all } i \\
\alpha a_{1}^{-1} \alpha=\alpha\left(a_{1}^{-1}\right)
\end{array}\right\rangle .
$$

We prove now that $G_{r, n} \cong A_{r, n}^{+} \rtimes \mathbb{Z}_{2}$. In order to do this, we define the following two homomorphisms, which are inverses of each other:

$$
\rho: A_{r, n}^{+} \rtimes \mathbb{Z}_{2} \rightarrow G_{r, n}
$$

defined on the generators by:

$$
\begin{aligned}
\alpha & \mapsto s_{0}^{\frac{r}{2}} \\
a_{0} & \mapsto s_{0}^{2} \\
a_{i} & \mapsto s_{0}^{\frac{r}{2}} s_{i} \text { for } i \geqslant 1,
\end{aligned}
$$


and

$$
\varphi: G_{r, n} \rightarrow A_{r, n}^{+} \rtimes \mathbb{Z}_{2}
$$

defined on the generators by:

$$
\begin{aligned}
s_{0} & \mapsto \alpha a_{0}^{\frac{r+2}{4}} \\
s_{i} & \mapsto \alpha a_{i} \text { for } i \geqslant 1 .
\end{aligned}
$$

It is easy to see that $\rho$ and $\varphi$ are isomorphisms, since $\rho \circ \varphi=I_{g_{r, n}}$ and $\varphi \circ \rho=I_{A_{r, n}^{+} \rtimes \mathbb{Z}_{2}}$, so we have that $G_{r, n} \cong A_{r, n}^{+} \rtimes \mathbb{Z}_{2}$.

Now, since $\rho\left(A_{r, n}^{+}\right) \subseteq A_{r, n}$ and $A_{r, n}, A_{r, n}^{+}$are both subgroups of $G_{r, n}$ of index 2 , they must be isomorphic.

The relations defining $A_{r, n}$ can be graphically described by the following Dynkinlike diagram, where the numbers inside the circles are the orders of the corresponding generators, an edge without a label between two circles means that the order of the multiplication of the two corresponding generators is 3 , and an edge labeled $2 r$ between two circles means that the order of the multiplication of the two corresponding generators is $2 r$ (two circles with no connecting edge mean that the two corresponding generators commute):

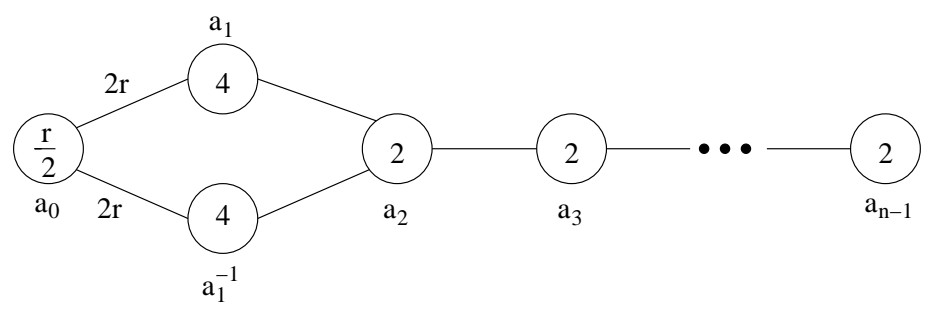

Figure 1: Dynkin-like diagram of $A_{r, n}$

\subsection{The length function}

Given a group $G$, generated by a set $A$, we denote by $\ell_{A}$ the length function on $G$ with respect to $A$. Explicitly, for each $\pi \in G$ :

$$
\ell_{A}(\pi)=\min \left\{u \mid g=a_{1} \cdots a_{u}, \text { where } a_{i} \in A\right\} .
$$

In this section, we prove that the algorithm described above, indeed gives us a reduced word with respect to the set of generators $\mathcal{A}$. In other words, we prove that for each $\pi \in A_{r, n}, \ell_{\mathcal{A}}(\pi)=L_{A}(\pi)$, where $L_{A}(\pi)$ is the number of generators in the presentation of $\pi$, obtained by the algorithm (and was computed in Theorem 13(2)).

We start with the following set of definitions: 


\section{Definition 17.}

- Let $\omega=b_{0} s_{0}^{i_{1}} b_{1} s_{0}^{i_{2}} b_{2} \cdots s_{0}^{i_{k}} b_{k} s_{0}^{i_{k+1}} b_{k+1}$ be any factorization of $\pi \in A_{r, n}$ as a product of the generators of $\mathcal{S}$, such that $b_{i} \in\left(\mathcal{S}-\left\{s_{0}\right\}\right)^{*}$ for $0 \leqslant i \leqslant k+1$.

- Let $n(\omega)$ be the number of generators $s_{i}$ in $\omega$ such that $i \neq 0$.

- Let $\beta(\omega)=\sum_{u=1}^{k+1}\left(i_{u} \oslash 2\right)$.

- Let $\mu(\pi)$ be the minimal value of $\beta(\omega)+n(\omega)$, obtained over all possible factorizations, $\omega$, of $\pi$ to $G_{r, n}$-generators as above.

Theorem 18. Let $\pi \in A_{r, n}$. Then: $\ell_{\mathcal{A}}(\pi)=\mu(\pi)$.

Proof. Let $\pi \in A_{r, n}$. We start by proving the inequality: $\ell_{\mathcal{A}}(\pi) \geqslant \mu(\pi)$. Let $\omega$ be a reduced word in generators from $\mathcal{A}$. Apply the map $\rho$, defined in the proof of Theorem 15 above, which sends $a_{i}$ to $s_{0}^{\frac{r}{2}} s_{i}$ for $i \in\{1, \ldots, n-1\}, a_{1}^{-1}$ to $s_{1} s_{0}^{\frac{r}{2}}$, and $a_{0}$ to $s_{0}^{2}$ on each letter separately, and concatenate.

This yields a word $\eta$, factorizing $\pi$ in $G_{r, n}$-generators, satisfying: $\ell_{\mathcal{A}}(\pi)=\beta(\eta)+n(\eta)$. This implies that the minimal value of $\mu$ over all the factorizations of $\pi$ to $G_{r, n}$-generators is at most $\ell_{\mathcal{A}}(\pi)$, since $\mu(\pi)$ is defined as the minimal value of all such expressions. So, we have: $\ell_{\mathcal{A}}(\pi) \geqslant \mu(\pi)$ (see Example 19(a) below).

In order to prove the opposite inequality: $\mu(\pi) \geqslant \ell_{A}(\pi)$, let $\omega$ be a factorization of $\pi$ to $G_{r, n}$-generators which achieves the minimal value of $\mu(\pi)$. Apply the map $\varphi$, defined in the proof of Theorem 15, which sends $s_{0}$ to $\alpha a_{0}^{\frac{r+2}{4}}$, and for all $i \geqslant 1$ sends $s_{i}$ to $\alpha a_{i}$ on each letter separately, and concatenate. This gives us a factorization $\eta$ of $\pi$ in $\mathcal{A} \cup\{\alpha\}$-generators, having $n(\omega)$ generators $a_{i}$ with $i \neq 0$ and $\beta(\omega)$ occurrences of $a_{0}$. The factorization $\eta$ contains also an even number of occurrences of the letter $\alpha$ (one occurrence for each $s_{i}$ for $i \geqslant 0$; recall that the number of $s_{i}$ 's is even by definition). By using the relations $\alpha a_{i} \alpha=a_{i}$ for $0 \leqslant i \leqslant n, i \neq 1$ and $\alpha a_{1}^{\varepsilon} \alpha=a_{1}^{-\varepsilon}$ for $\varepsilon \in\{ \pm 1\}$, we can cancel out all occurrences of $\alpha$ and we have an $\mathcal{A}^{*}$-word factorization of $\pi$ of length $\mu(\pi)$ (see Example 19(b) below). This proves that $\mu(\pi) \geqslant \ell_{\mathcal{A}}(\pi)$, since the length is the minimal number of generators of any presentation of $\pi$.

\section{Example 19.}

(a) We illustrate the proof of the first direction of the above proof: Let

$$
\pi=\left(1^{[5]} 3^{[3]} 2^{[3]} 4^{[0]}\right) \in A_{6,4} .
$$

The $\mathcal{A}^{*}$-word $w=a_{0} a_{1}^{-1} a_{2} a_{1} a_{2} a_{1}^{-1}$ is a reduced factorization of $\pi$. Apply the map $\rho$ on $\omega$ to get:

$$
\eta=s_{0}^{2} s_{1} s_{0}^{3} s_{0}^{3} s_{2} s_{0}^{3} s_{1} s_{0}^{3} s_{2} s_{1} s_{0}^{3} .
$$


One can calculate that:

$$
\begin{aligned}
\beta(\eta)+n(\eta) & =(2 \oslash 2)+(6 \oslash 2)+(3 \oslash 2)+(3 \oslash 2)+(3 \oslash 2)+5= \\
& =1+0+0+0+0+5=6=\ell_{\mathcal{A}}(\pi) .
\end{aligned}
$$

(b) In this part, we illustrate the derivation described in the second direction of the above proof: Let $\pi=\left(1^{[2]} 2^{[0]} 4^{[0]} 3^{[1]}\right)$, and let $\omega=s_{0}^{2} s_{2} s_{1} s_{0} s_{1} s_{2} s_{3}$ be a factorization of $\pi$ (here $\beta(\omega)+n(\omega)=8$ is indeed minimal). Then:

$$
\varphi(\omega)=\alpha a_{0}^{2} \alpha a_{0}^{2} \alpha a_{2} \alpha a_{1} \alpha a_{0}^{2} \alpha a_{1} \alpha a_{2} \alpha a_{3}=\eta .
$$

Now, after canceling out the appearances of $\alpha$, we get the $\mathcal{A}^{*}$-word:

$$
a_{0}^{2} a_{0}^{2} a_{2} a_{1} a_{0}^{2} a_{1} a_{2} a_{3}=a_{0} a_{2} a_{1} a_{0}^{2} a_{1} a_{2} a_{3}
$$

whose length is 8 too.

Theorem 20. Let $\pi \in A_{r, n}$. Then:

$$
L_{A}(\pi)=\mu(\pi)
$$

Proof. Let $\pi \in A_{r, n}$. The canonical decomposition of $\pi$ demonstrates the inequality $L_{A}(\pi) \geqslant \mu(\pi)$. For the opposite inequality, assume to the contrary that there is some decomposition $\omega$ of $\pi$ satisfying:

$$
n(\omega)+\beta(\omega)<L_{A}(\pi) .
$$

This contradicts the fact that Equation (5) is the length of $\pi$ with respect to the usual generators of $G_{r, n}$.

Hence, we have obtained the following corollary, which is the main result of this section:

Corollary 21. The function $L_{A}$ is indeed the length function of the group of alternating colored permutations with respect to the set of generators $\mathcal{A}$. Explicitly: for each $\pi \in A_{r, n}$,

$$
\ell_{\mathcal{A}}(\pi)=L_{A}(\pi)
$$

Consequently, we can easily get the generating function for the length function:

Corollary 22. The generating function for the length function $\ell_{\mathcal{A}}$ is:

$$
\sum_{\pi \in A_{r, n}} q^{\ell_{\mathcal{A}}(\pi)}=\frac{1}{2}[n] !_{q} \prod_{j=1}^{n}\left(1+q^{j-1}\left(1+2 q+\cdots+2 q^{\frac{r}{2}-1}\right)\right) .
$$


Proof. Each $\pi \in A_{r, n}$ has a canonical decomposition into generators from the set $\mathcal{S}$. By Theorem 4.4 of [2], the generating function for the length with respect to the set $\mathcal{S}$ over the whole group $G_{r, n}$ is:

$$
\sum_{\pi \in G_{r, n}} q^{\ell_{\mathcal{S}}(\pi)}=[n] !_{q} \prod_{j=1}^{n}\left(1+q^{j-1}\left(1+q+\cdots+q^{r-1}\right)\right) .
$$

By Theorem 13(1), the factor $s_{j-1} \cdots s_{1} s_{0}^{z}$ which colors the digit $j$ by $z$ colors is converted to $a_{j-1} \cdots a_{1}^{ \pm 1} a_{0}^{z \oslash 2}$. Since the mapping $\mathbb{Z}_{r} \rightarrow \mathbb{Z}_{\frac{r}{2}}$ is a $2: 1$-epimorphism, the factor $\left(1+q^{j-1}\left(1+q+\cdots+q^{r-1}\right)\right)$ is converted to $\left(1+q^{j-1}\left(1+2 q+\cdots+2 q^{\frac{r}{2}-1}\right)\right)$. Finally, after completing the coloring part, only half of the permutations of $S_{n}$ are permitted (since the total length of the word in $G_{r, n}$-generators should be even), so we have to divide the generating function by 2 .

\section{The group of alternating colored permutations as a covering group}

In [15], a covering map $f: A_{n+1} \rightarrow S_{n}$ was defined and used to lift some identities of $S_{n}$ to $A_{n+1}$. In this section, we use a similar technique with a covering map from $A_{r, n}$ to $G_{\frac{r}{2}, n}$. Unlike the case of $A_{n+1}$, this map is an epimorphism, and hence the kernel of this map will be combinatorially described. We also present a section $s: G_{\frac{r}{2}, n} \rightarrow A_{r, n}$ which gives us a way to decompose the length function, $\ell_{\mathcal{A}}(\pi)$, into two summands, one of them is constant on the coset of $\pi$, while the other, which will be called the fibral length, varies over the coset. We present a nice combinatorial interpretation of the last parameter, as well as a generating function for it over each coset. In Section 7, we use this covering map to lift some identities and permutation statistics from $G_{\frac{r}{2}, n}$ to $A_{r, n}$.

Define the following projection:

$$
p: A_{r, n} \rightarrow G_{\frac{r}{2}, n}
$$

as follows: if $\pi=\left(b_{1}^{\left[c_{1}\right]} \cdots b_{n}^{\left[c_{n}\right]}\right)$, then:

$$
p(\pi)=\left(b_{1}^{\left[c_{1} \oslash 2\right]} \cdots b_{n}^{\left[c_{n} \oslash 2\right]}\right) .
$$

Example 23. Let $\pi=\left(3^{[0]} 2^{[1]} 4^{[2]} 1^{[3]}\right) \in A_{6,4}$. Then:

$$
p(\pi)=\left(3^{[0 \oslash 2]} 2^{[1 \oslash 2]} 4^{[2 \oslash 2]} 1^{[3 \oslash 2]}\right)=\left(3^{[0]} 2^{[2]} 4^{[1]} 1^{[0]}\right) \in G_{3,4} .
$$

Then, we have:

Lemma 24. The map $p$ is an epimorphism. Moreover, the kernel of $p$ is the normal closure of $a_{1}^{2}$ in $A_{r, n}$. Thus: $G_{\frac{r}{2}, n} \cong \frac{A_{r, n}}{\ll a_{1}^{2} \gg}$. 
Proof. The map $p$ is clearly a homomorphism since the operator $\oslash$ commutes with the addition operation in $\mathbb{Z}_{\frac{r}{2}}$ (see Equation (4)). Now, if $\sigma=\left(b_{1}^{\left[c_{1}\right]} \cdots b_{n}^{\left[c_{n}\right]}\right) \in G_{\frac{r}{2}, n}$, and $j \in\{1, \ldots, n\}$ satisfies $b_{j}=1$, then, by Theorem 14, we have either

$$
\left(b_{1}^{\left[2 c_{1}\right]} \cdots b_{j}^{\left[2 c_{j}\right]} \cdots b_{n}^{\left[2 c_{n}\right]}\right) \in A_{r, n} \text { or }\left(b_{1}^{\left[2 c_{1}\right]} \cdots b_{j}^{\left[2 c_{j}+\frac{r}{2}\right]} \cdots b_{n}^{\left[2 c_{n}\right]}\right) \in A_{r, n}
$$

where the computations are made modulo $r$. This implies that $p$ is indeed an epimorphism.

It remains to find the kernel. Since $a_{1}^{2}=\left(1^{\left[\frac{r}{2}\right]} 2^{\left[\frac{r}{2}\right]} 3^{[0]} \cdots n^{[0]}\right)$, we have $a_{1}^{2} \in \operatorname{ker}(p)$, thus $\ll a_{1}^{2} \gg \leqslant \operatorname{ker}(p)$. On the other hand, all the elements of $\operatorname{ker}(p)$ are of the form $\left(1^{\left[c_{1}\right]} 2^{\left[c_{2}\right]} \cdots n^{\left[c_{n}\right]}\right)$, where $c_{i} \in\left\{0, \frac{r}{2}\right\}$ and $\left\{i \mid c_{i} \neq 0\right\} \mid$ is even. For each $i<j$, we can use the element $t_{i, j} a_{1}^{2} t_{i, j}^{-1} \in \ll a_{1}^{2} \gg$, where $t_{i, j}=s_{i-1} s_{i-2} \cdots s_{1} \cdot s_{j} s_{j-1} \cdots s_{2}$ in order to color digits $i$ and $j$ in $\frac{r}{2}$ colors without touching the other digits. This proves that $\ll a_{1}^{2} \gg=\operatorname{ker}(p)$, as needed.

We emphasize the following two observations, which can be concluded from the proof of the previous lemma, for a future use.

Observation 25. 1. $|\operatorname{ker}(p)|=2^{n-1}$.

2. Let $\pi, \pi^{\prime} \in A_{r, n}$ be such that $p(\pi)=p\left(\pi^{\prime}\right)$. Then, for each $i \in\{1, \ldots, n\}, c_{i}(\pi) \equiv$ $c_{i}\left(\pi^{\prime}\right)\left(\bmod \frac{r}{2}\right)$. Moreover, $c_{i}(\pi)$ and $c_{i}\left(\pi^{\prime}\right)$ differ by $\frac{r}{2}$ for an even number of indices.

The following obvious lemma presents the action of $p$ on the generators of $A_{r, n}$ :

\section{Lemma 26.}

$$
\begin{aligned}
& p\left(a_{i}\right)=s_{i} \text { for } 1 \leqslant i \leqslant n-1, \\
& p\left(a_{1}^{-1}\right)=s_{1}, \\
& p\left(a_{0}\right)=s_{0} .
\end{aligned}
$$

We introduce the following section of the covering map $p$ : Define

$$
s: G_{\frac{r}{2}, n} \rightarrow A_{r, n}
$$

as follows: if $\pi=\left(p_{1}^{\left[c_{1}\right]} \cdots p_{j}^{\left[c_{j}\right]} \cdots p_{n}^{\left[c_{n}\right]}\right)$ and $p_{j}=1$, then:

$$
\pi_{0}=s(\pi)=\left\{\begin{array}{cc}
\left(p_{1}^{\left[2 c_{1}\right]} \cdots p_{j}^{\left[2 c_{j}\right]} \cdots p_{n}^{\left[2 c_{n}\right]}\right) & \operatorname{inv}(|\pi|) \equiv 0(\bmod 2) \\
\left(p_{1}^{\left[2 c_{1}\right]} \cdots p_{j}^{\left[2 c_{j}+\frac{r}{2}\right]} \cdots p_{n}^{\left[2 c_{n}\right]}\right) & \operatorname{inv}(|\pi|) \equiv 1(\bmod 2),
\end{array}\right.
$$

where the computations are made modulo $r$. It is easy to verify that $p \circ s=\mathrm{Id}$.

Example 27. Let $\pi=\left(2^{[1]} 3^{[0]} 4^{[1]} 1^{[2]}\right) \in G_{3,4}$. Then, $\operatorname{inv}(|\pi|)=3$ and $j=4$. Thus, $\left(2^{[2]} 3^{[0]} 4^{[2]} 1^{[4]}\right) \notin A_{6,4}$, so $s(\pi)=\left(2^{[2]} 3^{[0]} 4^{[2]} 1^{[1]}\right) \in A_{6,4}$. 


\subsection{The fibral length}

For each $\pi \in A_{r, n}$, the length function of $\pi$ with respect to the set of generators $\mathcal{A}$ can be decomposed into two summands. The first summand is the length of $p(\pi)$ as an element in $G_{\frac{r}{2}, n}$, which is obviously invariant on the fiber of $\pi$. The second summand, which varies along the fiber, will be called the fibral length. As will be shown in this section, it has a nice combinatorial interpretation. We start with the definition of the fibral length.

Definition 28. For each $\pi \in A_{r, n}$, define the fibral length of $\pi$ to be:

$$
\ell_{F}(\pi)=\ell_{\mathcal{A}}(\pi)-\ell_{\mathcal{A}}(s(p(\pi)))
$$

For $\pi \in G_{r, n}$, denote $c(\pi)=\sum_{z_{i}(\pi) \neq 0}(i-1)$. By the definition of $\pi_{0}=s(p(\pi))$, we have:

$$
c(\pi)-c\left(\pi_{0}\right)=\sum_{\left\{i \mid z_{i}(\pi)=\frac{r}{2}\right\}}(i-1) .
$$

We will need the following two lemmata in the sequel:

\section{Lemma 29.}

$$
\ell_{F}(\pi)=c(\pi)-c\left(\pi_{0}\right)+\operatorname{inv}(\pi)-\operatorname{inv}\left(\pi_{0}\right) .
$$

Proof. Let $\pi \in A_{r, n}$. Then, by Observation 25(2),

$$
\sum_{i=1}^{n}\left(z_{i}(\pi) \oslash 2\right)=\sum_{i=1}^{n}\left(z_{i}\left(\pi_{0}\right) \oslash 2\right) .
$$

By Theorem 13(2), we are done.

Lemma 30. For each $\pi \in A_{r, n}$, we have: $\ell_{F}(\pi) \geqslant 0$.

Proof. Let $\pi \in A_{r, n}$. By Lemma 29, we have:

$$
\ell_{F}(\pi)=c(\pi)-c\left(\pi_{0}\right)+\operatorname{inv}(\pi)-\operatorname{inv}\left(\pi_{0}\right) .
$$

By Equation $(7), c(\pi)-c\left(\pi_{0}\right) \geqslant 0$. Now, let $1 \leqslant k<m \leqslant n$ be such that $\pi(k)=$ $i^{[\alpha]}<j^{[\beta]}=\pi(m)$, but $\pi_{0}(k)=i^{\left[\alpha^{\prime}\right]}>j^{\left[\beta^{\prime}\right]}=\pi_{0}(m)$. A rather tedious calculation should convince the reader that the only possibility is $|\pi(k)|=i>j=|\pi(m)|$ with $\alpha=\frac{r}{2}$ and $\beta=0$ (and hence $\alpha^{\prime}=\beta^{\prime}=0$ ), so that the digit $i$ is colored in $\pi$ but not in $\pi_{0}$. Now, since this situation can occur at most $i-1$ times, the contribution of $i$ to $c(\pi)-c\left(\pi_{0}\right)$ which is $i-1$, will cancel the corresponding negative contribution to $\operatorname{inv}(\pi)-\operatorname{inv}\left(\pi_{0}\right)$, and hence the total sum will be positive.

In order to make the previous proof a bit more accessible, we provide an example.

Example 31. Let $\pi=\left(3^{[3]} 21\right) \in A_{3,4}$, so that $\pi_{0}=(321)$. Then $i=3$ contributes 2 to $c(\pi)-c\left(\pi_{0}\right)$ and -2 to $\operatorname{inv}(\pi)-\operatorname{inv}\left(\pi_{0}\right)$ since $3^{[3]}<2,3^{[3]}<1$, but $3>1,3>2$. So, the total sum remains positive. 
Denote by $\ell_{G}$ the length function of the group $G_{\frac{r}{2}, n}$. Then, we have:

Lemma 32. Let $\pi \in A_{r, n}$ and $\pi_{0}=s(p(\pi))$. Then:

$$
\ell_{\mathcal{A}}\left(\pi_{0}\right)=\ell_{G}(p(\pi)) \text {. }
$$

Proof. Let $\pi \in A_{r, n}$. Then:

$$
\begin{aligned}
\left.\ell_{G}(p) \quad \ell(\pi)\right) & =\sum_{z_{i}(p(\pi)) \neq 0}(i-1)+\operatorname{inv}(p(\pi))+\sum_{i=1}^{n} z_{i}(p(\pi))= \\
& =\sum_{z_{i}(\pi) \notin\left\{0, \frac{r}{2}\right\}}(i-1)+\operatorname{inv}(p(\pi))+\sum_{i=1}^{n}\left(z_{i}(\pi) \oslash 2\right) .
\end{aligned}
$$

Now, $\sum_{z_{i}(\pi) \notin\left\{0, \frac{r}{2}\right\}}(i-1)=\sum_{z_{i}\left(\pi_{0}\right) \neq 0}(i-1)$, since for all $1<i \leqslant n, z_{i}\left(\pi_{0}\right) \neq \frac{r}{2}$. Moreover, one can check case-by-case that $\operatorname{inv}(p(\pi))=\operatorname{inv}\left(\pi_{0}\right)$.

Finally, one can check that for each $1 \leqslant i \leqslant n$, one has: $z_{i}\left(\pi_{0}\right) \oslash 2=z_{i}(\pi) \oslash 2$ since $z_{i}\left(\pi_{0}\right)-z_{i}(\pi) \in\left\{0, \frac{r}{2}\right\}$, and thus $\sum_{i=1}^{n}\left(z_{i}(\pi) \oslash 2\right)=\sum_{i=1}^{n}\left(z_{i}\left(\pi_{0}\right) \oslash 2\right)$.

Therefore, we have:

$$
\begin{aligned}
\ell_{G}(p(\pi)) & \stackrel{(*)}{=} \sum_{z_{i}(\pi) \notin\left\{0, \frac{r}{2}\right\}}(i-1)+\operatorname{inv}(p(\pi))+\sum_{i=1}^{n}\left(z_{i}(\pi) \oslash 2\right)= \\
& =\sum_{z_{i}\left(\pi_{0}\right) \neq 0}(i-1)+\operatorname{inv}\left(p\left(\pi_{0}\right)\right)+\sum_{i=1}^{n}\left(z_{i}\left(\pi_{0}\right) \oslash 2\right)=\ell_{\mathcal{A}}\left(\pi_{0}\right),
\end{aligned}
$$

as required.

As a corollary, we now have by Definition 28 and Lemma 32:

Corollary 33. Let $\pi \in A_{r, n}$ and let $p(\pi)$ be its projection into $G_{\frac{r}{2}, n}$. Then:

$$
\ell_{\mathcal{A}}(\pi)=\ell_{F}(\pi)+\ell_{G}(p(\pi)) .
$$

\subsection{A combinatorial interpretation of the fibral length}

For presenting the fibral length in a combinatorial way, we introduce the following parameter on $A_{r, n}$.

Definition 34. For each $\pi \in A_{r, n}$, define the set of absolute transparent inversions by:

$$
\operatorname{Tinv}(\pi)=\left\{(i, j) \mid z_{i}(\pi)=\frac{r}{2}, i>j, \text { and }|\pi|^{-1}(i)<|\pi|^{-1}(j)\right\} .
$$

Define also:

$$
\operatorname{tinv}(\pi)=|\operatorname{Tinv}(\pi)| .
$$


Example 35. If $\pi=\left(2^{[2]} 4^{[4]} 3^{[3]} 1^{[5]}\right) \in A_{6,4}$, then $\operatorname{Tinv}(\pi)=\{(3,1)\}$, since $\left(3^{[3]}, 1^{[5]}\right)$ is an absolute transparent inversion. Hence, $\operatorname{tinv}(\pi)=1$.

We have now:

Theorem 36. Let $\pi \in A_{r, n}$. Then:

$$
\ell_{F}(\pi)=2 \cdot \sum_{\left\{i \mid z_{i}(\pi)=\frac{r}{2}\right\}}(i-1)-2 \operatorname{tinv}(\pi) .
$$

Proof. By Equation (7) and Lemma 29, it is sufficient to show that:

$$
c(\pi)-c\left(\pi_{0}\right)=\operatorname{inv}(\pi)-\operatorname{inv}\left(\pi_{0}\right)+2 \operatorname{tinv}(\pi) .
$$

Let $1<i \leqslant n$ be such that $z_{i}(\pi)=\frac{r}{2}$. Then, by Equation (7), the contribution of $i$ to the left hand side is $i-1$, so we have to show that $i$ contributes the same to the right hand side, i.e. for each $1 \leqslant j<i$, the pair $(i, j)$ contributes 1 to the expression $\operatorname{inv}(\pi)-\operatorname{inv}\left(\pi_{0}\right)+2 \operatorname{tinv}(\pi)$. This can be easily done by a subtle, though, direct check. Note that $i=1$ contributes 0 to both sides.

If $1 \leqslant i \leqslant n$ satisfies $z_{i}(\pi) \neq \frac{r}{2}$, then $i$ contributes 0 to both sides.

We are interested in the distribution of the fibral length of $\pi \in A_{r, n}$ over the coset containing $\pi$. Define:

$$
F(\pi)=\sum_{\sigma \in p^{-1}(p(\pi))} q^{\ell_{F}(\sigma)} .
$$

It would be much easier to calculate this distribution if we translate Theorem 36 to the language of Lehmer codes [13]. Recall that the Lehmer code of a permutation $\pi \in S_{n}$ is defined by:

$$
L(\pi)=\left(l_{\pi(1)} \cdots l_{\pi(n)}\right)
$$

where for each $1 \leqslant i \leqslant n$,

$$
l_{i}=\left|\left\{j \mid j>i, \pi^{-1}(i)>\pi^{-1}(j)\right\}\right| .
$$

For example, if $\pi=(31452) \in S_{5}$ (in window notation), then:

$$
L(\pi)=\left(l_{3} l_{1} l_{4} l_{5} l_{2}\right)=(20110) .
$$

Note that this definition is slightly different from the usual definition of the Lehmer code.

For $\pi \in A_{r, n}$, let $L(|\pi|)=\left(l_{1} \cdots l_{n}\right)$, and define:

$$
\varepsilon_{i}(\pi)=\left\{\begin{array}{cc}
1 & z_{i}(\pi)=\frac{r}{2} \\
0 & z_{i}(\pi) \neq \frac{r}{2}
\end{array}\right.
$$

The parameter $\operatorname{tinv}(\pi)$ can be written as:

$$
\operatorname{tinv}(\pi)=\sum_{i=1}^{n} l_{i} \varepsilon_{i}(\pi)
$$


and Equation (8) can be restated as:

$$
\ell_{F}(\pi)=2 \sum_{i=1}^{n} \varepsilon_{i}(\pi)\left((i-1)-l_{i}\right) .
$$

The following theorem presents an expression for the generating function $F(\pi)$, using Equation (10).

Theorem 37. Let $\pi \in A_{r, n}$ and define for each $2 \leqslant i \leqslant n$ :

$$
\delta_{i}(\pi)=\left\{\begin{array}{cc}
1 & z_{i}(\pi) \in\left\{0, \frac{r}{2}\right\} \\
0 & z_{i}(\pi) \notin\left\{0, \frac{r}{2}\right\}
\end{array}\right.
$$

Then:

$$
F(\pi)=\prod_{i=2}^{n}\left(1+q^{2 \delta_{i}\left(i-1-l_{i}\right)}\right) .
$$

Proof. Let $\pi=\left(a_{1}^{\left[c_{1}\right]} \cdots a_{n}^{\left[c_{n}\right]}\right) \in A_{r, n}$. By Observation 25(2), the coset of $\pi$ is

$$
\left\{\left(a_{1}^{\left[d_{1}\right]} \cdots a_{n}^{\left[d_{n}\right]}\right)\right\}
$$

where the vector $\left(d_{1}, \ldots, d_{n}\right)$ is obtained from the vector $\left(c_{1}, \ldots, c_{n}\right)$ by an addition of $\frac{r}{2}$ to an even number of coordinates. Hence, the vectors $\left(d_{1}, \ldots, d_{n}\right)$, appearing as colors of elements of the coset of $\pi$ can be seen as forming the $(n-1)$-dimensional affine subspace $\left(c_{1}, \ldots, c_{n}\right)+S p\left(\left\{e_{1}-e_{2}, \ldots, e_{n-1}-e_{n}\right\}\right)$, where $e_{i}=\left(0, \ldots, \frac{r}{2}, \ldots, 0\right)$ (i.e. $\frac{r}{2}$ in the $i$-th coordinate and 0 elsewhere). Finally, note that when we run over all the elements of the coset, only the coordinates with $z_{i}(\pi) \in\left\{0, \frac{r}{2}\right\}$ and $i \neq 1$ contribute to $\ell_{F}(\pi)$.

\section{$7 \quad$ Some permutation statistics}

In this section, we present some permutation statistics for the group of alternating colored permutations.

\subsection{Passing parameters from $G_{\frac{r}{2}, n}$ to $A_{r, n}$}

We exhibit now how to pass parameters defined on the full group of colored permutations of half the number of colors to the group of alternating colored permutations. In order to do that, we have to define the notion of a fiber-fixed parameter.

Definition 38. Let $f_{A}: A_{r, n} \rightarrow \mathbb{N}$ and $f_{K}: G_{\frac{r}{2}, n} \rightarrow \mathbb{N}$ be two permutation statistics. The parameter $f_{A}$ is called fiber-fixed if for each $\pi \in A_{r, n}$,

$$
f_{K}(p(\pi))=f_{A}(\pi)
$$


By Observation 25(1), we have the following connection between the corresponding generating functions:

Lemma 39. Let $f_{A}: A_{r, n} \rightarrow \mathbb{N}$ and $f_{K}: G_{\frac{r}{2}, n} \rightarrow \mathbb{N}$ be such that $f_{A}$ is a fiber-fixed parameter. Then:

$$
\sum_{\pi \in A_{r, n}} q^{f_{A}(\pi)}=2^{n-1} \sum_{\pi \in G_{\frac{r}{2}, n}} q^{f_{K}(p(\pi))} .
$$

Proof.

$$
\begin{gathered}
\sum_{\pi \in A_{r, n}} q^{f_{A}(\pi)}=\sum_{\pi \in G_{\frac{r}{2}, n}} \sum_{v \in p^{-1}(\pi)} q^{f_{A}(v)}=\sum_{\pi \in G_{\frac{r}{2}, n}} \sum_{v \in p^{-1}(\pi)} q^{f_{K}(p(v))}= \\
=2^{n-1} \sum_{\pi \in G_{\frac{r}{2}, n}} q^{f_{K}(\pi)} .
\end{gathered}
$$

We give two examples of fiber-fixed parameters, the first one is the flag-inversion number, and the second is the right-to-left minimum.

\subsection{The flag-inversion number}

The flag-inversion number was introduced by Foata and Han [9, 10]. Adin, Brenti and Roichman [1] used it as a rank function for a weak order on the groups $G_{r, n}$. We introduce it in $G_{r, n}$ :

Definition 40. Let $\pi \in G_{r, n}$. The flag-inversion number of $\pi$ is defined as:

$$
\operatorname{finv}(\pi)=r \cdot \operatorname{inv}(|\pi|)+\operatorname{csum}(\pi) .
$$

In [11], the generating function of finv over $G_{r, n}$ was computed:

\section{Proposition 41.}

$$
\sum_{\pi \in G_{r, n}} q^{\mathrm{finv}(\pi)}=\prod_{i=1}^{n}[r i]_{q} .
$$

We define here a version of the flag-inversion number for the alternating colored permutations whose generating function over $A_{r, n}$ can be computed using on the covering $\operatorname{map} p$.

Definition 42. Let $\pi \in A_{r, n}$. Define:

$$
\operatorname{finv}_{A}(\pi)=\frac{r}{2} \cdot \operatorname{inv}(|\pi|)+\sum_{i=1}^{n}\left(c_{i}(\pi) \oslash 2\right) .
$$

It is easy to see that the parameter finv is indeed fiber-fixed, in the sense of Equation (11). Explicitly, for each $\pi \in A_{r, n}, \operatorname{finv}_{A}(\pi)=\operatorname{finv}(p(\pi))$, since $|\pi|=|p(\pi)|$ and $c_{i}(\pi) \oslash 2$ is fixed on each fiber. Consequently, we have: 
Theorem 43.

$$
\sum_{\pi \in A_{r, n}} q^{\mathrm{finv}_{A}(\pi)}=2^{n-1} \prod_{i=1}^{n}\left[\frac{r}{2} \cdot i\right]_{q} .
$$

\subsection{The right-to-left minima}

Another parameter, which can be computed using the covering map, is the right-to-left minima.

Definition 44. Let $p=\left(a_{1}, \ldots, a_{n}\right)$ be a word over an ordered alphabet $(\Sigma,<)$. Then $a_{i} \in\{1, \ldots, n\}$ is a right-to-left minimum if for any $j>i$, one has: $a_{j}>a_{i}$. The number of right-to-left minima will be denoted by $\operatorname{RtlMin}(p)$.

Example 45. Let $p=(31254) \in S_{5}$ (with the natural order), then 1,2,4 are right-to-left minima and hence: $\operatorname{RtlMin}(p)=3$.

Regev and Roichman [16] defined a version of the right-to-left minima for $G_{r, n}$ as follows:

Definition 46. Let $\pi=\left(a_{1}^{\left[c_{1}\right]} \cdots a_{n}^{\left[c_{n}\right]}\right) \in G_{r, n}$. Define:

$$
\operatorname{RtlMin}(\pi)=\left|\left\{a_{i} \mid \forall j>i: a_{j}>a_{i}, c_{i} \neq 0\right\}\right| \text {. }
$$

They showed that the distribution of the parameter RtlMin over the full group of colored permutations is (see Proposition 5.1 in [16] with $L=\{0, \ldots, r-1\}$ ):

$$
\sum_{\pi \in G_{r, n}} q^{\operatorname{RtlMin}(\pi)}=((r-1) q+1)((r-1) q+r+1) \cdots((r-1) q+(n-1) r+1) .
$$

We introduce here a version of the right-to-left minima for $A_{r, n}$.

Definition 47. Let $\pi \in A_{r, n}$. Define:

$$
\operatorname{RtlMin}_{A}(\pi)=\left|\left\{a_{i} \mid \forall j>i, a_{j}>a_{i}, c_{i} \neq\left\{0, \frac{r}{2}\right\}\right\}\right| .
$$

Again, it is easy to see that RtlMin ${ }_{A}$ is fiber-fixed. Explicitly, for each $\pi \in A_{r, n}$, we have $\operatorname{RtlMin}_{A}(\pi)=\operatorname{RtlMin}(p(\pi))$, since we are considering only digits whose colors are not 0 or $\frac{r}{2}$. Hence, as an immediate corollary of Proposition 5.1 of [16], we get:

Theorem 48.

$$
\sum_{\pi \in A_{r, n}} q^{\mathrm{RtIMin}_{A}(\pi)}=2^{n-1} \prod_{i=1}^{n}\left(\frac{r}{2}(q+i-1)+1-q\right) .
$$

\section{Acknowledgments}

We would like to thank Yuval Roichman for fruitful discussions. 


\section{References}

[1] R.M. Adin, F. Brenti and Y. Roichman, Flag weak order on wreath products, Semii. Lothar. Combin., 67, 2012, Art. B67e.

http://www.mat. univie.ac.at/ slc/wpapers/s67adberoi.pdf

[2] E. Bagno, Euler-Mahonian parameters on colored permutation groups, Semí. Lothar. Combin., 51, 2004, Art. B51f.

http://www.mat.univie.ac.at/ slc/wpapers/s51bagno.pdf

[3] E. Bagno and D. Garber, On the excedance number of colored permutation groups, Semii. Lothar. Combin., 53, 2006, Art. B53f.

http://www . mat. univie.ac.at/ slc/wpapers/s53bagngarb.pdf

[4] N. Bourbaki, Lie Groups and Lie Algebras, English translation by Andrew Pressley, Springer, 2002.

[5] A. Björner and F. Brenti, Combinatorics of Coxeter groups, Grad. Texts in Math., Vol. 231, Springer-Verlag, 2005.

[6] M. Broué, G. Malle and R. Rouquier, Complex reflection groups, braid groups, Hecke algebras, J. Reine Angew. Math., 500:127-190, 1998.

[7] F. Brenti, V. Reiner and Y. Roichman, Alternating subgroups of Coxeter groups, J. Combin. Theory Ser. A, 115(5):845-877, 2008.

[8] J. Désarménien and D. Foata, Statistiques d'ordre sur les permutations colorées, Discrete Math., 87(2):133-148, 1991.

[9] D. Foata and G.-N. Han, Signed words and permutations. I. A fundamental transformation, Proc. Amer. Math. Soc., 135:31-40, 2007.

[10] D. Foata and G.-N. Han, Statistical distributions on words and q-calculus on permutations, Strasbourg, 2007.

[11] M. Fire, Statistics on wreath products, Preprint. arXiv:math/0409421

[12] J.E. Humphreys, Reflection groups and Coxeter groups, Cambridge University press, 1992.

[13] D.H. Lehmer, Teaching combinatorial tricks to a computer, Proc. Sympos. Appl. Math. Combinatorial Analysis, 10:179-193, 1960.

[14] A. Niedermaier and J. Remmel, Analogues of up-down permutations for colored permutations, J. Integer Seq., 13(5), 2010, Article 10.5.6, 32 pp.

[15] A. Regev and Y. Roichman, Permutation statistics on the alternating group, Adv. Appl. Math., 33(4):676-709, 2004.

[16] A. Regev and Y. Roichman, Statistics on wreath products and generalized binomialStirling identities, Israel J. Math., 151:189-221, 2006.

[17] E. Steingrimsson, Permutation statistics of indexed permutations, European J. Combin., 15:187-205, 1994. 